\title{
Enhancing the Ostrom social-ecological system framework through formalization
}

\author{
$\underline{\text { Jochen Hinkel }}^{1}, \underline{\text { Pieter W. G. Bots }}^{2}$ and Maja Schlüter $^{3}$
}

\begin{abstract}
Frameworks play an important role in analyzing social-ecological systems (SESs) because they provide shared concepts and variables that enable comparison between and accumulation of knowledge across multiple cases. One prominent SES framework focusing on local resource use has been developed by Elinor Ostrom and her colleagues. This framework is an extensive multi-tier collection of concepts and variables that have demonstrated relevance for explaining outcomes in a large number of case studies in the context of fishery, water, and forestry common-pool resources. The further development of this framework has raised a number of issues related to the formal relationships between the large number of concepts and variables involved. In particular, issues related to criteria for ordering the concepts into tiers, adding new concepts, defining outcomes metrics, and representing dynamics in the framework have been identified. We address these issues by applying methods from research fields that study formal relationships between concepts such as domain-specific languages, knowledge representation, and software engineering. We find that SES frameworks could include the following seven formal components: variables, concepts, attribution relationships, subsumption relationships, process relationships, aggregation relationships, and evaluation metrics. Applying these components to the Ostrom framework and a case study of recreational fishery, we find that they provide clear criteria for structuring concepts into tiers, defining outcome metrics, and representing dynamics. The components identified are generic, and the insights gained from this exercise may also be beneficial for the development of other SES frameworks.
\end{abstract}

Key Words: formalization; framework; ontology; SES; social-ecological system; taxonomy

\section{INTRODUCTION}

The development of frameworks is a central activity in the study of social-ecological systems (SESs) because frameworks provide scholars from different backgrounds common languages for comparing between and building theory across cases (Ostrom 2009). Given that SESs are complex and multi-level systems (Berkes and Folke 1998) studied at all scales and within diverse contexts, multiple frameworks are available and indeed necessary for understanding different aspects of SESs (Poteete et al. 2010, Binder et al. 2013). These include, for example, the SES vulnerability framework (Turner et al. 2003) and resilience thinking (Folke et al. 2010), to name just two. See Binder et al. (2013) for a comparison of these and other SES frameworks. The variety of frameworks available also reflects the variety of disciplines and research fields involved in the study of SESs, including sustainability science, landscape ecology, ecological economics, geography, resource economics, and resilience thinking, which all contribute different and valuable perspectives on social-ecological interactions and outcomes.

One prominent effort at developing a framework to analyze SESs in the context of local communities sharing a common resource has been taken by Ostrom and colleagues (Ostrom 2007, 2009, McGinnis and Ostrom 2014). The Ostrom framework builds on the institutional analysis and development framework (Ostrom 2005), but also draws strongly from the literature on the resilience of SESs (e.g., Berkes and Folke 1998). It is a multi-tier collection of concepts and variables that have been proven useful for understanding resource use in the context of fishery, water, and forestry common-pool resources. On the first tier, the framework conceptualizes SESs into resource systems, resource units, governance systems, actors, interactions, and outcomes. These higher-tier concepts are then decomposed into more fine-grained lower-tier concepts and variables (Fig. 1). The first-tier concept resource units, for example, is decomposed into second-tier variables such as resource unit mobility, replacement rate, economic value, and size.

The Ostrom framework has been constructed incrementally over decades through the empirical analyses of a large number of case studies. Concepts and variables that appeared common across cases were collected and added to the framework. Framework development is ongoing in the context of the so-called SES Club, a group of scholars, including the authors of this paper, Elinor Ostrom, and others (McGinnis and Ostrom 2014). This further development and application of the Ostrom framework in the wider literature has raised a number of issues regarding the formal relationships between concepts and variables of the framework. Among these are:

- What exactly is the meaning of the tiers, and where should new concepts and variables be added to the framework (McGinnis and Ostrom 2014)?

- How can nested levels of ecological and social aggregation be analyzed? The empirical analysis of ecosystems, for example, often requires to the collection of data on the level of individual organisms as well as on the level of populations (Schlüter et al. 2014). Furthermore, the Ostrom framework is ambiguous as to whether some of the variables attached to actors refer to the individual or the collective level ( $\mathrm{J}$. Hinkel et al., unpublished manuscript).

- How do the variables listed under outcomes (e.g., resilience, sustainability, robustness) relate to the other variables in the framework? 
Fig. 1. The first-tier and second-tier concepts of the Ostrom (2009) social-ecological system framework, including minor refinements made by McGinnis and Ostrom (2014).

Social, Economic, and Political Settings (S)

S1- Economic development. S2- Demographic trends. S3- Political stability. S4- Other governance systems.

S5-Markets. S6-Media organizations. S7-Technology.

Resource Systems (RS)

Governance Systems (GS)

RS1- Sector (e.g., water, forests, pasture, fish)

RS2- Clarity of system boundaries

RS3- Size of resource system

RS4- Human-constructed facilities

RS5- Productivity of system

RS6- Equilibrium properties

RS7- Predictability of system dynamics

RS8- Storage characteristics

RS9- Location

Resource Units (RU)

GS1- Government organizations

GS2- Nongovernment organizations

GS3- Network structure

GS4-Property-rights systems

GS5-Operational-choice rules

GS6-Collective-choice rules

GS7-Constitutional-choice rules

GS8-Monitoring and sanctioning rules

Actors (A)

RU1- Resource unit mobility

RU2- Growth or replacement rate

RU3- Interaction among resource units

RU4-Economic value

RU5- Number of units

RU6- Distinctive characteristics

RU7-Spatial and temporal distribution

A1- Number of relevant actors

A2- Socioeconomic attributes

A3- History or past experiences

A4- Location

A5- Leadership/entrepreneurship

A6- Norms (trust-reciprocity)/social capital

A7-Knowledge of SES/mental models

A 8- Importance of resource (dependence)

A9- Technologies available

Action Situations: Interactions $(\mathrm{I}) \rightarrow$ Outcomes $(\mathrm{O})$

Activities and Processes:

I1- Harvesting

I2- Information sharing

13- Deliberation processes

14- Conflicts

15- Investment activities

16- Lobbying activities

17- Self-organizing activities

18- Networking activities

19- Monitoring activities

I10- Evaluative activities
Outcome Criteria:

01-Social performance measures

(e.g., efficiency, equity, accountability, sustainability)

O2- Ecological performance measures

(e.g., overharvested, resilience, biodiversity, sustainability)

O3- Externalities to other SESs

Related Ecosystems (ECO)

ECO1- Climate patterns. ECO2- Pollution patterns. ECO3- Flows into and out of focal SES.

- How can dynamics and interactions between variables be represented (Schlüter et al. 2014; U. J. Frey and E. Ostrom, unpublished manuscript)? SESs are dynamical systems with variables that influence each other. The Ostrom framework lists some of these interaction (e.g., self-organizing activities or information sharing), but does not specify which lowertier variables are involved.

Here, we address these issues through formalization. The issues listed above are common to framework development and have been addressed in research fields that study formal relationships between concepts such as domain specific languages, knowledge representation, and software engineering. We explore to what extent the further development of the Ostrom framework may benefit from applying insights and methods from these fields. The purpose of this exercise is to improve the clarity of the description of the Ostrom framework. Formalization per se does not aim at making any changes to the framework. It does, however, provide a basis for extending and generalizing the framework by providing clear criteria for adding new concepts and refining existing ones. The components and criteria identified are generic and may also be valuable for the development of other SES frameworks.

Formalization involves trade-offs. On the one hand, formalization may enhance comparability and foster accumulation of knowledge on SESs by providing for a more structured and comparable analysis. Formalization is also a precondition for the development of databases for large-N studies, which has been a topic for several years in the context of the Ostrom framework (e.g., Poteete and Ostrom 2008). On the other hand, formalization is time and resource intensive, and an over-formalized framework may be counter-productive for understanding SESs because it may force the analyst into one particular perception of SESs and make him/ 
her blind to other aspects that might be more important (Hinkel 2008). A lower degree of formalization also makes the framework more accessible for newcomers, and its concepts may better serve as boundary concepts for facilitating interdisciplinary dialog (Mollinga 2010).

Here, we first review the basic ideas of framework development and formalization, which we then apply to explore what kind of formal components an SES framework might include. We then apply these findings to refine the Ostrom framework, and test this refinement on a case of a recreational fishery for which we have first-hand knowledge. Finally, we reflect on our approach and provide some conclusions.

\section{FRAMEWORKS AND FORMALIZATION}

A framework is a domain-specific language or "little language", which is a language tailored for a specific knowledge domain (Bently 1986, Abelson and Sussman 1987, Hudak 1998). The elements of a framework are concepts (i.e., terms associated with a meaning) and conceptual relationships between them. Imagine, for example, a simple framework for the domain of forestry. Such as framework might contain concepts such as "tree", "conifer", "oak", and "trunk", as well as conceptual relationships such "a conifer is a tree" and "a tree has a trunk".

Generally, the purpose of developing a shared framework is to facilitate the exchange of arguments about the knowledge domain through having a clear and unambiguous account of the concepts at stake and their relationships. In the domain of SESs, this means that a framework should support the formulation and comparison of hypotheses, models, and theories. An SES framework thus aims to provide the most general set of concepts that is assumed to be applicable to the entire domain of SESs (Ostrom 2005). Although this does not mean that a framework is free of theory, the goal of framework development is to stay as neutral as possible to allow the representation of different theories within the framework.

The idea of formalization is closely related to the one of framework development. Formalization is the process of making form (or structure) of linguistic expressions explicit by translating them into a formal language (Hinkel 2008, Ionescu et al. 2009, Wolf et al. 2013). As suggested by the name, formalization targets form, that is, the relationships between concepts rather than the meaning of the concepts themselves. Hence, any framework that makes relationships between concepts explicit is, by definition, to some extend formal. Many frameworks, for example, express the relationships between concepts through the formal language of box-and-arrow diagrams. However, there are also many other formal languages available, including other graphical languages, mathematics, and programming languages.

Generally, formalization is a common activity in research. Scholars frequently formalize text into a table or box-and-arrow diagram. The degree of formalization usually increases in the evolution of scientific fields or disciplines (Bertalanffy 1968, Suppes 1968). This is a gradual process that may go through several stages, starting with the extension of the ordinary language lexicon through the introduction of technical terms, the standardization of the syntax of the language, the replacement of some technical language expressions through artificial symbols, and possibly, translation into mathematics (Posner 2003).
For the purpose of improving the clarity of the conceptual relationships in the Ostrom framework, we draw from the existing literature on formal languages. Two sets of formal languages are of particular importance. First, because SESs are dynamical systems, languages for representing influence or causal relationships between concepts and variables are important. Prominent examples are causal loop, stock-and-flow (Forrester 1961), and influence diagrams (Howard and Matheson 2005), as well as mathematical language in general.

Second, because the tiers of the Ostrom framework relate concepts semantically, languages that represent semantic relationships between concepts are relevant. One prominent example originating from the field of software engineering is the unified modeling language (e.g., Fowler and Scott 1997). In environmental sciences, the unified modeling language has, for example, been applied to develop a framework for analyzing water policy regimes (Pahl-Wostl et al. 2010). Other prominent examples originating from the field of artificial intelligence are knowledge representation languages. These languages date back to original work on existential graphs (Peirce 1909), semantic networks and frames (Minsky 1975), and description logics (Baader and Nutt 2003). A prominent contemporary representative is the ontology web language (e.g., McGuinness and Harmele 2004). The word ontology is used here, similar to the word framework, as a formal description of concepts, objects, and relationships among those of a given knowledge domain (Gruber 1993). In environmental natural sciences, ontologies are, for example, applied for improving and automating data and model integration (e.g., Madin et al. 2007, Villa 2007, Villa et al. 2009).

A treatment of these formal languages involves technicalities and would be beyond the scope of this paper. Here, we limit ourselves to describing informally those components relevant for SES framework development in general and the Ostrom framework in particular. Readers interested in technical aspects of formalizing the Ostrom framework are referred to Appendix 1, where we develop a simple formal notation called METAFRAME that captures the components relevant here.

\section{COMPONENTS OF A SOCIAL-ECOLOGICAL SYSTEM FRAMEWORK}

Variables and data

The basic components of any framework used in empirical analysis are variables. Variables are a special kind of concepts that can take on different values (Bernard 2000). The variables "Precipitation" and "Biomass", for example, could take on positive real numbers. The specific values recorded for variables at a given point in space and time are called data.

\section{Concepts and attribution relationships}

Whenever empirical analysis involves many variables, as is usually the case for SESs, it is useful to organize them semantically; that is, variables are grouped according to their meaning. One common way of doing this is by attributing variables to higher-level concepts. An attribute is "a quality or feature regarded as a characteristic or inherent part of someone or something" (Oxford Dictionary 2010). The semantic relationship between higher-level concepts and its attributes is called attribution or "has-a" relationship. In the simple forestry framework mentioned above, 
for example, the variables "Biomass" and "EconomicValue" may be attributes of "Forest".

When concepts are grouped together with further variables into higher-level concepts, an attribution hierarchy is attained. Expanding our simple forestry framework, we may take "SES" as the top concept of the hierarchy and attribute "Forest", "User", and "Environment" to it (Fig. 2). "Biomass" occurs twice in the hierarchy: once as an attribute of "Forest" and once as an attribute of "Tree". To distinguish between these, we use the following dot notation: "Tree.Biomass" refers to the biomass of a single tree, whereas "Forest.Biomass" refers to the biomass of an entire forest.

Fig. 2. A simple forestry example of a social-ecological system (SES) framework. Boxes denote concepts, black arrows pointing down denote attribution relationships, black open arrowheads pointing up denote subsumption relationships, brown links denote aggregation relationships. A 1 indicates a one-to-one attribution relationship; an asterisk indicates a oneto-many attribution relationship. Concept names are indicated in boldface in the top part of the boxes; attributed variables are listed in the bottom part of the boxes. Process relationships and outcome metrics are not shown.



\section{Subsumption relationships}

Another prominent way of organizing concepts semantically is by subsumption. Because the goal of an SES framework is to be applicable to different kinds of SESs, it must be possible to express that one concept generalizes or specializes another one. For example, it may be desirable to express that "forest is a special kind of resource system" or "conifer is a special kind of tree". This kind of semantic relationship is called subsumption or "isa" relationship (also known as hyponym-hypernym, inheritance, generalization-specialization, or sub-concept-super-concept relationship). It links a more general concept called super-concept to a more specific concept called sub-concept.

The recursive grouping of concepts by subsumption relationships leads to a subsumption hierarchy, which is also called taxonomy, typology, or classification. This idea goes back to the method of Aristotle to define concepts in terms of their genus proximum (nearest genus, here super-concept) and differentia specifica (specific difference). For example, the Oxford Dictionary (2010) defines a conifer as a tree (genus) that "bears cones and needlelike or scale-like leaves that are typically evergreen" (specific difference). Prominent historical examples of taxonomies are the
Porphyrian tree (Porphyry 1992) and the Linnaean taxonomy of species (von Linné and Lange 1770).

\section{Aggregation relationships}

A third kind of semantic relationship that SES frameworks might include is the aggregation relationship between variables. SESs are nested, multi-level systems, which means that data for variables are often collected, calculated, or simulated on different levels of aggregation. In the simple forestry framework, for example, we have represented biomass at the level of individual trees as well as at the level of the whole forest. The latter can be though of as the sum of the former. Other common aggregation relationships are count, average, and standard deviation. Because the meaning of these functions is well-defined using mathematical language, aggregation relationships are an efficient means to unambiguously define the meaning of an aggregate variable in terms of other variables.

\section{Outcome metrics}

The fourth kind of semantic relationship that might be useful in SES frameworks is outcome metrics. These are used because complex outcomes such as sustainability, resilience, stability, and welfare are not directly observable but must be computed by or indicated through a set of other variables of the SES framework. In our simple forestry framework, for example, we could think of an outcome metric called sustainability that is computed through the biomass of the forest as well as the harvest of a user. We symbolize this as

Sustainability $\leftarrow$ (Forest.Biomass, User.Harvest),

where " $\leftarrow$ " means "is indicated through". Formally, an outcome metric can be thought of as a function that relates a set of directly measurable variables with a theoretical variable standing for a concept such as sustainability, resilience, stability, or welfare (Hinkel 2011).

\section{Process relationships}

Because the purpose of SES framework application is to understand which variables interact in determining outcomes, it may be desirable to capture influence or process relationships between variables. The process by which the variable "Precipitation" influences "Biomass", for example, could be called "Growth". Using the right arrow " $\rightarrow$ " to denote that the variables on the left hand side influence the variable on the right hand side, we depict this as

Growth: Precipitation $\rightarrow$ Biomass.

It is important to note that process relationships are a different type of relationship than the semantic relationships. They differ in that they are irrespective of the linguistic meaning of the variables themselves. Saying that "precipitation influences biomass" does not relate the two concepts semantically, but only relates values of the former variable to values of the later.

Because SESs are dynamical systems, a special kind of process of particular importance is feedback processes. These are processes in which values of variables at one point in time influence their own values at a future point in time (Kalman et al. 1969). For example:

\section{Growth: (Biomass, Precipitation) $\rightarrow$ Biomass.}

From the perspective of framework development, it is desirable to represent those processes that are general to all cases. The most 
Fig. 3. The refined Ostrom social-ecological system (SES) framework. Boxes denote concepts, black arrows pointing down denote attribution relationships, black open arrowheads pointing up denote subsumption relationships, brown links denote aggregation relationships. A 1 indicates a one-to-one attribution relationship; an asterisk indicates a one-to-many attribution relationship. Concept names are indicated in boldface in the top part of the boxes; attributed variables are listed in the bottom part of the boxes. Process relationships and outcome metrics are not shown.

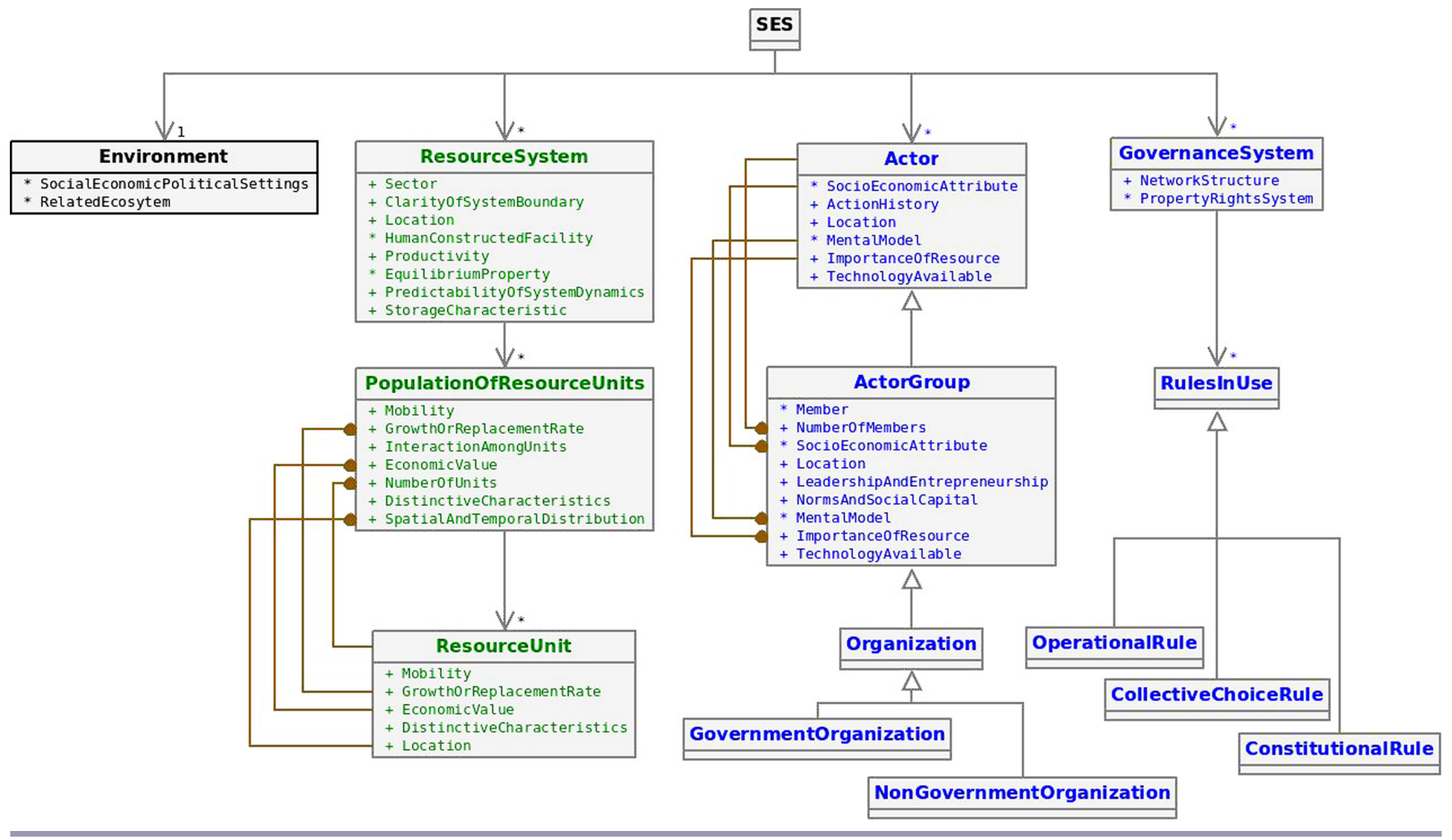

general process applicable to all SESs is the global transition of the SES, that is, the process that may involve all variables of the SES. Because the concept SES is, as explained above, a variable composed of all variables entailed in its attribution hierarchy, we can represent the global transition as

Transition: SES $\rightarrow$ SES.

This formula can be seen as a shorthand notation for referring to all the attributes of the concept SES. It can thus be unpacked through replacing each higher-level concept by the list of its attributes until one ends up with all the variables, that is, concepts that cannot be decomposed further. For example:

Transition: (SES.Forest, SES.User, SES.Environment) $\rightarrow$ (SES. Forest, SES.User, SES.Environment)

Transition: (SES.Forest.Biomass, SES.Forest.EconomicValue, SES.Forest.Tree.Biomass, SES.User.Harvest, SES.User.Technology, SES.Environment.Precipitation) $\rightarrow$ (SES.Forest.Biomass, SES. Forest.EconomicValue, SES.Forest.Tree.Biomass, SES.User. Harvest, SES.User.Technology, SES.Environment.Precipitation).

The expanded representation of the transition process, however, may include variables that do not influence the dynamics of the SES; the goal of analysis is to be as specific as possible by getting rid of variables that neither influence the process nor are influenced by it. In our simple forestry framework, we could thus specialize the transition process to:
Transition: (SES.Forest.Biomass, SES.User.Harvest, SES. Environment.Precipitation) $\rightarrow$ SES.Forest.Biomass.

This completes the exploration of components relevant for an SES framework. Our example shows the complete forestry framework attained (Fig. 2).

\section{APPLYING FRAMEWORK COMPONENTS TO THE OSTROM FRAMEWORK}

We now apply the seven components identified above to the Ostrom framework to test how formalization can help to resolve the ambiguities that have been encountered by researchers, and those we listed in Introduction, when applying the Ostrom framework (Fig. 3).

\section{Meaning of the tiers and where to add new variables}

The tiers of the Ostrom framework can be formalized as attribution and subsumption relationships in a straightforward manner. This results in four first-tier concepts attributed to the top-level concept SES (Fig. 3): An SES "has" (i.e., attribution relationship) resource systems, governance systems, actors, and an environment. One main difference to the structure of the Ostrom framework is that ResourceUnits (RU) does not appear on the first tier but on the second tier. Also, Interactions (I) and Outcomes $(\mathrm{O})$ are not included in this semantic hierarchy because these are better thought of as metrics and process relationships. We will come back to this below. The two remaining first-tier 
concepts, SocialEconomicAndPoliticalSettings (S) and RelatedEcosystems (ECO), are attributed to the concept "Environment" because these are, by definition, external to the focal SES analyzed.

Subsumption relationships appear further down the tiers under the concepts Actors (A) and GoverenceSystems (GS). OperationalRules, CollectiveChoiceRules, and ConstitutionalRules are subsumed under the concept Rules-in-use. Similarly, GovernanceOrganizations and NongovernmentalOrganizations are subsumed under Organizations.

The subsumption and attribution hierarchies also give clarity on where to add new variables. The attribution relation " $\mathrm{X}$ has-a $\mathrm{Y}$ " may be applied only if the English phrase " $\mathrm{X}$ has a $\mathrm{Y}$ " is grammatically correct and meaningful for all instances of $X$ and Y. A new variable $Y$ that (at first glance) seems to represent a property of some concept $\mathrm{X}$ may be attributed to $\mathrm{X}$ only if it cannot be attributed to a super-concept of X. For example, the property ActionHistory of A should not be attributed to ActorGroups but rather to its super-concept A because it applies to actors in general. Regarding subsumption, the subsumption relation " $\mathrm{X}$ is-a Z" may be applied only if the English phrase "all $\mathrm{Xs}$ are Zs (but not vice versa)" is a true statement. For example, all Organizations are ActorGroups, and all ActorGroups are A (but not all $\mathrm{A}$ are ActorGroups because an actor may also be an individual).

\section{Analyzing nested levels of ecological aggregation}

The Ostrom framework contains two first-tier concepts related to the ecological system, namely the resource system and resource units. Semantically, resource units, however, are part of resource systems. The fish (resource unit) a fisher extracts from a fish population are part of the lake or coastal water (resource system) it lives in. We therefore attribute Ostrom's first-tier concept RU to the other first-tier concept ResourceSystems (RS) because a resource system can be meaningfully thought of as having units.

Additionally, we split Ostrom's RU into the two concepts, PopulationOfResourceUnits and ResourceUnits, and attribute the latter to the former because some of the attributes of Ostrom's RU refer to populations (or collections) of resource units, whereas others refer to individual units. Most attributes may, however, refer to both concepts. Hence, we attribute them to both and connect them by aggregation relationships. The "Economic value" (RS4) of a population of resource units, for example, may be thought of as an attribute that aggregates economic values of all resource units. Two attributes of Ostrom's RU, "Interaction among resource units" (RS3) and "Spatial and temporal distribution" (RS7) only make sense for populations of units are are thus attributed only to this concept. The latter is formalized as an aggregated variable of the Location of individual RU.

\section{Analyzing nested levels of social aggregation}

Similarly to the case of RU, we see the need to introduce explicitly a micro-macro distinction for Ostrom's first-tier variable A because some variables of A describe attributes of groups of actors (e.g., number of users, norms, and social capital), whereas others may describe both groups of actors as well as single actors (e.g., history of use, socioeconomic attributes, and importance of resource). Moreover, in heterogeneous groups, the values of these latter variables may differ between actors of the same group.

We therefore take the concept Actors as the top-level concept denoting abstractly any social entity that acts (e.g., individuals, groups, collective actors, organizations, etc.) and attach all variables that may describe any actors to it. Next, we introduce ActorGroups as a sub-concept of $\mathrm{A}$ and attach a variable Members, which refers to all the actors that are members of the group. Group members may be either individual actors or actor groups, which allows representation of nested levels of societal organization. Then, we formalize the variable "number of users" as NumberOfMembers by means of an aggregation relation with A.

Next, we duplicate some of the variables that have also been attributed to $\mathrm{A}$. This time, however, we introduce them by means of aggregation relationships with their corresponding variables on the level of $\mathrm{A}$. We attribute those variables that can only be used for describing a group of actors (e.g, number of users, leadership/entrepreneurship, and norms/social capital) to ActorGroups. Finally, we attach Ostrom's concepts "government and non-government organizations" (GS1 and GS2) to the actor hierarchy rather than to the governance system, as the Ostrom framework does, because organizations are special kinds (i.e., subconcepts) of actor groups. Future work needs to elaborate these concepts in terms of what kind of variables are relevant for describing them.

\section{Institutions and nested levels of social aggregation}

In the context of the Ostrom framework, institutions are understood as formal and informal rules that shape human interactions (North 1991). With regard to formalizing this, we encountered the challenge of weather to give a subsumption or attribution hierarchy presidency. The first option would mean to introduce a concept Institution and distinguish different kinds of institutions. Crawford and Ostrom (1995), for example, have developed such a subsumption hierarchy that draws a distinction between rules, which are institutions that include a sanctioning mechanisms, and norms, which are institutions that do not. The Ostrom framework, however, does not make use of this subsumption hierarchy but follows a second option and attributes norms to actors (i.e., a group of actors has norms) and rules to the governance system (i.e., a governance system has rules).

We also decided to follow this second option because, for the purpose of explaining outcomes in local common-pool resource use, it is important to distinguish between norms being internal to a group of actors and rules that may be both internally developed or externally imposed on the actors (McGinnis and Ostrom 2014). For the rules, we then introduce a subsumption hierarchy that distinguishes between the different types of rules (i.e., operational rules, collective-choice rules, and constitutional rules).

\section{Relating outcomes to other variables}

Variables listed under Ostrom's first-tier concept Outcomes such as Equity, Efficiency, Sustainability, and Resilience can be interpreted in a straightforward manner as outcome metrics because these are complex measures of the state of the SES. However, the Ostrom framework does not provide information on which variables of RS, GS, RU, and A are related to these outcome metrics. This is understandable because the set of variables used to indicate, e.g., resilience, may vary from case to case. On the other hand, to achieve the long-term goal of developing theory from multiple cases, it would be desirable to select common metrics to be used across cases. One step toward this end would be to list, for each outcome metric, the variables 
that influence this metric. To give an example, an outcome metric called resilience could be defined as a function of the number of resource units, their growth rates, and equilibrium properties:

Resilience $\leftarrow$ (NumberOfUnits, GrowthOrReplacementRate, EquilibriumProperty).

\section{Representing dynamics in the framework}

The names given to the variables collected under Ostrom's firsttier concept Interactions suggest that these should be interpreted as processes, rather than as variables (e.g., information sharing, deliberation processes, and self-organizing activities). Similarly, ActionSituations (AS) are social and environmental processes through which interactions lead to outcomes. However, the Ostrom framework does not provide information on which lowertier variables are involved in these processes. It only represents what was called earlier the global transition process of the SES:

Transition: (Environment, GovernanceSystem, Actor, ResourceSystem) $\rightarrow$ (GovernanceSystem, Actor, ResourceSystem).

Understanding outcomes and dynamics of SESs consists precisely in further decomposing and characterizing this global transition process. The variety of interactions, action situations, and also, biophysical processes covered by the Ostrom framework could be made more precise by listing the concepts and variables that influence or are influenced by these processes. We give some directions that future work may take.

Ostrom (2005), for example, classifies action situations as constitutional-choice, collective-choice, and operational-choice situations. This classification is based on which variables are influenced by the respective action situations or processes. A constitutional choice process influences the value of the variable CollectiveChoiceRule, a collective choice process influences the variableOperationalRule, and an operational choice process influences one or more variables attributed to the concept RS. Symbolizing other relevant variables referring to social, political, economic, biophysical, and legal context as “...", these processes could be represented as

ConstitutionalChoice: (Actors, ...) $\rightarrow$ CollectiveChoiceRule, CollectiveChoice: (Actors, CollectiveChoiceRule, ...) $\rightarrow$ OperationalRule,

OperationalChoice: (Actors, OperationalChoiceRule, ...) $\rightarrow$ ResourceSystem.

The classification of processes or action situations would then proceed by further unfolding the concepts involved. At the operational level, for example, action situations may be distinguished into production, appropriation, distribution, consumption, evaluation, monitoring, and sanctioning. At the collective-choice level, it would be interesting to distinguish between self-organized collective choice processes (SelfOrganization) and ones in which the government crafts the operational rules (Regulation):

SelfOrganization: (Users, CollectiveChoiceRule) $\rightarrow$ OperationalRule, Regulation: (GovernmentOrganization, CollectiveChoiceRule) $\rightarrow$ OperationalRule.

\section{CASE STUDY OF A RECREATIONAL FISHERY}

Here, we test whether the formalized Ostrom framework is likewise robust when we try to accommodate the variety of variables used in a detailed empirical study of an SES. As a first test, we apply the formal components to a specific SES case study from a recreational fishery of which we have first-hand knowledge (Fig. 4). In a second step, we compare the results with the formalized Ostrom framework. See also Schlüter et al. (2014), who elaborate this case with a particular focus on representing the dynamics of SESs through process relationships.

Fig. 4. The social-ecological system (SES) framework applied to the case of a recreational fishery. Boxes denote concepts, black arrows pointing down denote attribution relationships, black open arrowheads pointing up denote subsumption relationships, brown links denote aggregation relationships. A 1 indicates a one-to-one attribution relationship; an asterisk indicates a one-to-many attribution relationship. Concept names are indicated in boldface in the top part of the boxes; attributed variables are listed in the bottom part of the boxes. Process relationships and outcome metrics are not shown.

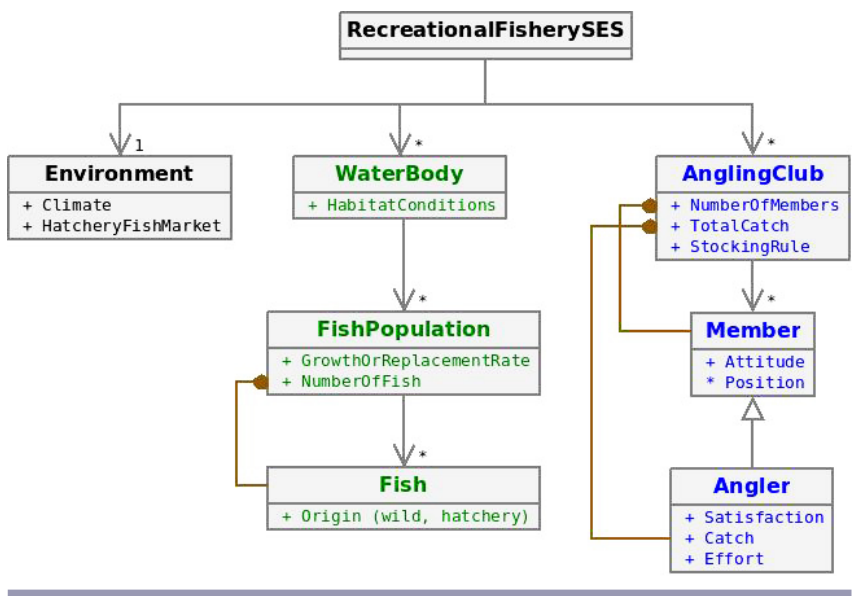

Recreational fisheries are the dominant users of freshwater and major coastal fish stocks in industrialized countries (Arlinghaus and Cooke 2009). In Germany, angling associations and local angling clubs regulate access and perform management activities in accordance with their fishing rights. They are thus responsible for the maintenance of the ecological integrity of water bodies in the face of angling exploitation. Stocking, that is, the introduction of fish from a hatchery or a different water body into an existing fish population, is one of the most commonly applied management measures. However, its economic efficiency, social implications, and ecological effects are often not known. Our formalization of a recreational fishery SES is therefore guided by the research question: Can stocking enhance the sustainability of recreational fisheries? It is thereby assumed that attributes of both the social system (e.g., angler preferences) and the ecological system (e.g., habitat conditions) of the recreational fisheries SES determine the outcome of stocking measures. Whether an outcome is considered sustainable is evaluated based on ecological, social, and economic indicators such as the replacement rate of wild fish by hatchery fish, the social welfare of the club, or the economic performance.

The top-level concept we apply is the recreational fishery SES. When delineating the recreational fishery SES, we must decide which concepts/variables are considered to be internal, that is, 
part of the focal SES, and which ones are considered to be external, that is, part of its environment (see also McGinnis and Ostrom 2014). This choice depends on the research question because variables are internal if they both influence and are influenced by the variables entailed by the research question. In the chosen case study, we consider variables such as angling club, members, and fish population to be internal because their interactions co-determine the values of the state variables and the indicators in which we are interested. Hence, we define the concept AnglingClub and the concepts WaterBody and FishPopulation as attributes of theRecreationalFisheriesSES. Concepts such as Climate and the HatcheryFishMarket are defined as external because we assume that they are not influenced by the variables of the focal SES. These are thus attributed to the Environment of the SES.

An AnglingClub has many Members, so we attribute the later to the former concept. Member has other variables attributed to it such as the Position of a member in a club and his/her Attitude toward stocking. Some members are anglers (so Angler is a subconcept of Member) who have additional attributes such as the effort put into angling (Effort) and the fish caught (Catch). Further attributes of the angling club may be the stocking rules (StockingRule), which prescribe the stocking activities, and TotalCatch, which aggregates the catch of all anglers.

When formalizing the fish population and the water body, which affects the survival and growth of the fish population, we faced the following two alternatives. We could either attribute WaterBody to the FishPopulation or we could organize it the other way around and define WaterBody as the higher-level concept with relevant variables attributed to it, FishPopulation being one of them. We chose the second option because the same water body can be populated by several fish populations, and it is more meaningful to say that "the water body has a fish population" than "the fish population has a water body".

With respect to the processes, we only give two examples here because these are treated in full detail in Schlüter et al. (2014). In a recreational fisheries SES that is enhanced by stocking, relevant processes relate to the exploitation of the fish population by anglers, and the growth of the fish population. These can be formalized by the following two processes relationships:

Angling: (Angler.Effort, FishPopulation) $\rightarrow$ Angler.Catch, FishGrowth: (FishPopulation.NumberOfFish, FishPopulation. GrowthOrReplacementRate, AnglingClub.TotalCatch) $\rightarrow$ FishPopulation. NumberOfFish.

In a second step, we position the concepts identified for this specific case (Fig. 4) into the hierarchy of the formalized Ostrom framework (Fig. 3). Many of the concepts identified as relevant for the fishery case could be easily incorporated as sub-concepts into the general SES framework. An AnglingClub, for example, is an ActorGroup; the WaterBody is a ResourceSystem, with the FishPopulation being a PopulationOfResourceUnits and a Fish being a ResourceUnit.

The fisheries framework, however, also contains variables that have not yet been included in the general SES framework. Many of these are located at the micro-level of human behavior, which is largely absent in the SES framework but may become of relevance in certain empirical investigations. Examples are psychological variables of individual actors such as Member. Attitude and Angler.Satisfaction. As these influence the actions of members, such as their angling and stocking behavior, they are relevant for stocking outcomes. The same is true for some attributes of the resource unit such as the origin of a fish from a hatchery or a wild fish population. Although these variables are new, fitting them into the formalized SES framework is a straightforward exercise, which gives some initial indication of the robustness of the formalization of the Ostrom framework elaborated here.

\section{CONCLUSION}

We applied concepts and methods from fields related to formalization and language development to resolve existing ambiguities in the Ostrom SES framework. Based on the literature, we identified the following seven formal components an SES framework might include: variables, concepts, attribution relationships, subsumption relationships, aggregation relationships, outcome metrics, and process relationships. We then applied these seven components to the Ostrom framework and a case study of a recreational fishery. Through this process, we were able to refine the Ostrom framework in a number of ways. We introduced subsumption hierarchies, in particular, for actors and rules-inuse. We also introduced a micro-macro distinction for both actors and the resource systems that allows the description of properties of individual actors and resource units as well as groups of actors and populations of resource units. Aggregation relationships link the corresponding variables between the two levels. We showed that the variables belonging to Ostrom's first-tier concept Outcomes are meaningfully thought of as outcome metrics that map values of a number of state variables to theoretical variables such as sustainability, resilience, or equity.

Another major refinement proposed is the representation of variables belonging to Ostrom's first-tier concepts Interactions and ActionSituation as process relationships. So far, the Ostrom framework does not specify which variables influence or are influenced by Interactions, nor does the framework include relevant biophysical processes. We have given some directions on how to include these processes and how to represent action situations in terms of the lower-tier variables involved. Future work needs to carry this refinement further through classifying processes without jeopardizing the generality of the framework. Of particular relevance are those processes that link actions of diverse resource users to outcomes (i.e., action situations) because these create interdependences between users; capturing these interdependencies is essential for understanding the emergence and design of governance structures for sustainable SESs (Anderies et al. 2004; J. Hinkel et al., unpublished manuscript).

The formalization of the Ostrom framework brings several benefits. It makes the analysis more consistent and makes it easier to relate the variables measured in the field to the variables in the framework because the formalized framework clearly expresses the semantic relationships between concepts (i.e., attribution, subsumption). For the same reason, this fosters the further development of the framework because new variables can be integrated more easily in the framework. The formalized framework also helps to decompose different scales and levels of aggregation (via aggregation relationship). Finally, it serves as a tool to define the variables that are involved in the action 
situations of interest (process relationships); particularly, the latter helps to communicate about the dynamics of an SES and may facilitate discussions on what theories/causal relationships are suitable. See Schlüter et al. (2014) for a further demonstration of this aspect.

Formalization and framework development, however, also come at a price. Both are resource and time intensive, and the trade-off between investing time/resources and the benefits of achieving greater rigor and comparability needs to be considered. Formalization is most useful when the application domain is narrow and well defined. Formalization was thus beneficial in the case of the Ostrom framework because it is grounded in a solid empirical basis that has been established over decades. Furthermore, the application domain of local communities managing forestry, fishery, and water resources is rather narrowly defined. When generalizing from this situation to more complex ones, the Ostrom framework may be less suitable and formalization less helpful (J. Hinkel et al., unpublished manuscript).

Finally, we emphasize that, similar to the Linnaean system, the advantages of SES frameworks are, of course, a function of their acceptance by SES researchers. Developing a shared framework can only be accomplished over a long period of time through collaboration, debate, and iterative refinement involving scholars that study different aspects of SESs in various contexts. Here, we contribute to this debate by providing directions for further developing the Ostrom framework.

Responses to this article can be read online at: http://www.ecologyandsociety.org/issues/responses. $\mathrm{php} / 6475$

\section{Acknowledgments:}

We thank Claudia Binder, Katrin Daedlow, Michael McGinnis, Elinor Ostrom, Alexander Bisaro, Ralf Seppelt, and three anonymous reviewers for their very helpful comments on earlier versions of this paper as well as all members of the SES Club for the many stimulating discussions we had about the issues this paper addresses.

\section{LITERATURE CITED}

Abelson, H., and G. J. Sussman. 1987. Lisp: a language for stratified design. Byte 13(2):207-218.

Anderies, J. M., M. A. Janssen, and E. Ostrom. 2004. A framework to analyze the robustness of social-ecological systems from an institutional perspective. Ecology and Society 9(1): 18. [online] URL: http://www.ecologyandsociety.org/vol9/iss1/art18/.

Arlinghaus, R. and S. J. Cooke. 2009. Recreational fisheries: socioeconomic importance, conservation issues and management challenges. Pages 39-58 in B. Dickson, J. Hutton, and W. M. Adams, editors. Recreational hunting, conservation and rural livelihoods: science and practice. Wiley-Blackwell, Chichester, UK. http://dx.doi.org/10.1002/9781444303179.ch3
Baader, F., and W. Nutt. 2003. Basic description logics. Pages 43-95 in F. Baader, D. Calvanese, D. McGuinness, D. Nardi, and P. Patel-Schneider, editors. The description logic handbook: theory, implementation and applications. Cambridge University Press, Cambridge, UK.

Bentley, J., 1986. Programming pearls: little languages. Communications of the ACM 29(8):711-721. http://dx.doi. org/10.1145/6424.315691

Berkes, F., and C. Folke. 1998. Linking social and ecological systems for resilience and sustainability. Pages 1-25 in F. Berkes and C. Folke, editors. Linking social and ecological systems: management practices and social mechanisms for building resilience. Cambridge University Press, Cambridge, UK.

Bernard, H. R. 2000. Social research methods: qualitative and quantitative approaches. Sage, Thousand Oaks, California, USA.

Bertalanffy, L. V. 1968. General system theory: foundations, development, applications. Revised edition. George Braziller, New York, New York, USA.

Binder, C. R., J. Hinkel, P. W. G. Bots, and C. Pahl-Wostl. 2013. Comparison of frameworks for analyzing social-ecological systems. Ecology and Society 18(4): 26. http://dx.doi.org/10.5751/ ES-05551-180426

Crawford, S. E. S., and E. Ostrom. 1995. A grammar of institutions. American Political Science Review 89(3):582-600. http://dx.doi.org/10.2307/2082975

Folke, C., S. R. Carpenter, B. Walker, M. Scheffer, T. Chapin, and J. Rockström. 2010. Resilience thinking: integrating resilience, adaptability and transformability. Ecology and Society 15(4): 20. [online] URL: http://www.ecologyandsociety.org/vol15/iss4/ $\underline{\operatorname{art} 20 /}$.

Forrester, J. W. 1961. Industrial dynamics. MIT Press, Cambridge, Massachusetts, USA.

Fowler, M., and K. Scott. 1997. UML distilled: applying the standard object modeling language. Addison Wesley Longman, Reading, Massachusetts, USA.

Gruber, T. R. 1993. A translation approach to portable ontology specifications. Knowledge Acquisition 5(2):199-220. http://dx.doi. org/10.1006/knac. 1993.1008

Hinkel, J. 2008. Transdisciplinary knowledge integration: cases from integrated assessment and vulnerability assessment. Dissertation. Wageningen University, Wageningen, The Netherlands. [online] URL: http://ciret-transdisciplinarity.org/ biblio/biblio pdf/Hinkel.pdf.

Hinkel, J. 2011. "Indicators of vulnerability and adaptive capacity": towards a clarification of the science-policy interface. Global Environmental Change 21(1):198-208. http://dx.doi. org/10.1016/j.gloenvcha.2010.08.002

Howard, R. A., and J. E. Matheson. 2005. Influence diagrams. Decision Analysis 2(3):127-143. http://dx.doi.org/10.1287/ deca.1050.0020

Hudak, P. 1998. Modular domain specific languages and tools. Pages 134-142 in Proceedings of the Fifth International Conference 
on Software Reuse. IEEE, Bangalore, India. http://dx.doi. org/10.1109/ICSR.1998.685738. http://dx.doi.org/10.1109/ ICSR.1998.685738.

Ionescu, C., R. J. T. Klein, J. Hinkel, K. S. K. Kumar, and R. Klein. 2009. Towards a formal framework of vulnerability to climate change. Environmental Modelling and Assessment 14 (1):1-16. http://dx.doi.org/10.1007/s10666-008-9179-x

Kalman, R. E., P. L. Falb, and M. A. Arbib. 1969. Topics in mathematical system theory. McGraw-Hill, New York, New York, USA.

Madin, J., S. Bowers, M. Schildhauer, S. Krivov, D. Pennington, and F. Villa. 2007. An ontology for describing and synthesizing ecological observation data. Ecological Informatics 2(3):279-296. http://dx.doi.org/10.1016/j.ecoinf.2007.05.004

McGinnis, M. D., and E. Ostrom. 2014. Social-ecological system framework: initial changes and continuing challenges. Ecology and Society, in press.

McGuinness, D. L., and F. van Harmele. 2004. OWL web ontology language: overview. W3C recommendation 10 February 2004. W3C, Massachusetts Institute of Technology, Cambridge, Massachusetts, USA. [online] URL: http://www.w3.org/ TR/2004/REC-owl-features-20040210.

Minsky, M. L. 1975. A framework for the representation of knowledge. Pages 211-277 in P. H. Winston, editor. The psychology of computer vision. McGraw-Hill, New York, New York, USA.

Mollinga, P. P. 2010. Boundary work and the complexity of natural resources management. Crop Science 50:S1-S9. http://dx. doi.org/10.2135/cropsci2009.10.0570

North, D. C. 1991. Institutions. Journal of Economic Perspectives 5(1):97-112. http://dx.doi.org/10.1257/jep.5.1.97

Ostrom, E. 2005. Understanding institutional diversity. Princeton University Press, Princeton, New Jersey, USA.

Ostrom, E. 2007. A diagnostic approach for going beyond panaceas. Proceedings of the National Academy of Sciences 104 (39):15181-15187. http://dx.doi.org/10.1073/pnas.0702288104

Ostrom, E. 2009. A general framework for analyzing sustainability of social-ecological systems. Science 325:419-422. http://dx.doi.org/10.1126/science.1172133

Oxford Dictionary. 2010. Oxford dictionary. Oxford University Press, Oxford, UK. [online] URL: http://oxforddictionaries.com.

Pahl-Wostl, C., G. Holtz, B. Kastens, and C. Knieper. 2010. Analyzing complex water governance regimes: the management and transition framework. Environmental Science \& Policy 13 (7):571-581. http://dx.doi.org/10.1016/j.envsci.2010.08.006

Peirce, C. S. 1909. Existential graphs: manuscript 514, with commentary by J. F. Sowa. Self-published by J. F. Sowa. [online] URL: http://www.jfsowa.com/peirce/ms514.htm.

Porphyry. 1992. On Aristotle's categories. Translated by S. K. Strange. Cornell University Press, Ithaca, New York, USA.

Posner, R. 2003. The semiotic reconstruction of individual disciplines. Pages 2563-2569 in R. Posner, K. Robering, and T. A. Sebeok, editors. Semiotics: a handbook on the sign-theoretic foundations of nature and culture. Volume 3. Walter de Gruyter, Berlin, Germany.

Poteete, A. R., M. A. Janssen, and E. Ostrom. 2010. Working together: collective action, the commons, and multiple methods in practice. Princeton University Press, Princeton, New Jersey, USA.

Poteete, A. R., and E. Ostrom. 2008. Fifteen years of empirical research on collective action in natural resource management: struggling to build large- $\mathrm{N}$ databases based on qualitative research. World Development 36(1):176-195. http://dx.doi. org/10.1016/j.worlddev.2007.02.012

Schlüter, M., J. Hinkel, P. W. G. Bots, and R. Arlinghaus. 2014. Application of the SES framework for model-based analysis of the dynamics of social-ecological systems. Ecology and Society 19(1): 36. http://dx.doi.org/10.5751/ES-05782-190136

Suppes, P. 1968. The desirability of formalization in science. Journal of Philosophy 65(20):651-664. http://dx.doi.org/10.2307/2024318

Turner, II, B. L., R. E. Kasperson, P. A. Matson, J. J. McCarthy, R. W. Corell, L. Christensen, N. Eckley, J. X. Kasperson, A. Luers, M. L. Martello, C. Polsky, A. Pulsipher, and A. Schiller. 2003. A framework for vulnerability analysis in sustainability science. Proceedings of the National Academy of Sciences 100 (14):8074-8079. http://dx.doi.org/10.1073/pnas.1231335100

Villa, F. 2007. A semantic framework and software design to enable the transparent integration, reorganization and discovery of natural systems knowledge. Journal of Intelligent Information Systems 29(1):79-96. http://dx.doi.org/10.1007/s10844-006-0032$\underline{x}$

Villa, F., I. N. Athanasiadis, and A. E. Rizzoli. 2009. Modelling with knowledge: a review of emerging semantic approaches to environmental modelling. Environmental Modelling \& Software 24(5):577-587. http://dx.doi.org/10.1016/j.envsoft.2008.09.009

von Linné, C., and J. J. Lange. 1770. Systema naturae: per regna tria naturae, secundum classes, ordines, genera, species, cum characteribus, differentiis, synonymis, locis. Volume 3. Curt.

Wolf, S., J. Hinkel, M. Hallier, A. Bisaro, D. Linke, C. Ionescu, and R. J. T. Klein. 2013. Clarifying vulnerability definitions and assessments using formalisation. International Journal of Climate Change Strategies and Management 5(1):54-70. http://dx.doi. org/10.1108/17568691311299363 


\section{Appendix}

\section{METAFRAME}

\subsection{Rationale}

Based on the seven formal components a SES framework identified in Section 3 of the main paper, we have developed a compact, human-readable textual notation, called METAFRAME. The goal of this development was to provide non-technically trained SES scholars with a simply human-readable syntax that can be easily used (and has been used) in textual form via emails to exchange ideas about SES framework design decisions such as adding new concept or variables. The notation represents a SES framework in the form of nested bullet points as this has been used before by SES scholars (McGinnis and Ostrom this issue). The notation emerged through interaction with the group of authors of this paper.

At the same time, parts of METAFRAME can be directly mapped to (and hence automatically translated into) ontology languages such as OWL. In the future, once sufficient clarity on the SES framework has been gained and datasets using the framework become available, this will facilitate implementing SES-related web resources using Semantic Web technologies. We illustrate the notation with the help of the simple forestry framework introduced in Section 3 of the main paper.

\subsection{Variables and data types}

In METAFRAME, we use the following data types for variables:

- "String": The set of all concatenations of symbols in the English alphabet plus punctuation symbols (i.e., the set of all natural language expressions).

- "Number": The set of all real numbers.

- " "[a,b]": The set of all real numbers $>=$ a and $<=b$.

- "Boolean": The set of logical constants (i.e., "\{true, false\}").

- "\{word1, word2, word3\}": arbitrary sets of strings/words.

To express that a variable has a certain data type, we simply place the type behind a colon that follows the variable name. For variables of type "Number", we specify the unit in which a quantity is expressed in square brackets. For example:

Biomass: Number [kg]

EconomicValue: Number [US\$]

Precipitation: Number [mm/yr]

\subsection{Concepts and attribution relationships}


We denote attributes as bullet points written below the concept to which they are attributed to. In the simple forestry framework, for example, the variables "Biomass" and

"EconomicValue" may be attributes of "Forest". We write this as:

Forest

+ Biomass: Number [kg]

+ EconomicValue: Number [US\$]

The " + " expresses a one-to-one attribution relationship (e.g., "A SES has one

Environment"), while the "* " expresses a one-to-many attribution relationship left (e.g., "A

SES has one or more Users"). When concepts are grouped together with further variables into higher-level concepts, an attribution hierarchy is attained. Expanding our simple forestry framework, we may take "SES" as the top concept of the hierarchy and attribute "Forest", "User" and "Environment" (everything external to the SES) to it.

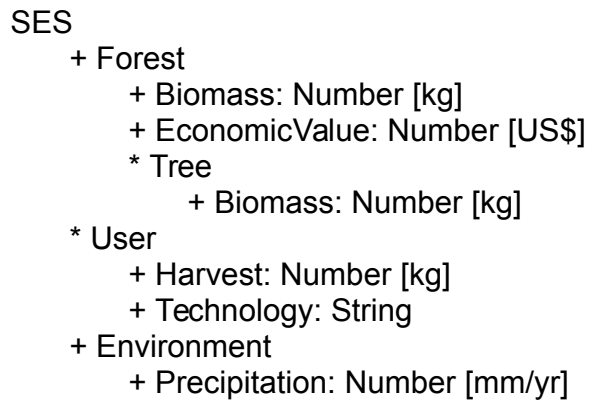

Since all concepts at the bottom of the attribution hierarchy are variables, the whole hierarchy can be seen as a complex variable of type SES. Just as one can associate a single value to a primitive variable (e.g., harvest $=40 \mathrm{~kg}$, or technology = "saw"), one can associate lists of values to the concepts, being complex variables (e.g., user $=(40 \mathrm{~kg}$, "saw")).

\subsection{Subsumption relationships}

We express the subsumption relationship, similarly to the graphical notation, as a nested bullet-point list using the symbol " $\wedge$ ". For example:



\subsection{Aggregation relationships}

Formally, aggregation relationships are mathematical functions that map atomic variables (variables that are not disaggregated further) to aggregate ones. In METAFRAME we represent this by writing the aggregation function behind the aggregate variable. For example: 
Forest

+ Biomass: Number $[\mathrm{kg}]=$ sum $($ Tree*.Biomass $)$

* Tree

+ Biomass: Number [kg]

Other common aggregation functions are count, average, and standard deviation.

\subsection{Outcome metrics}

In our simple framework, for example, we could think of an outcome metric called "sustainability" that is computed through the biomass of the forest as well as the harvest of a user. In METAFRAME we write this as:

Sustainability <- (Forest.Biomass, User.Harvest)

\subsection{Process relationships}

Formally, process relationships are mathematical relations on the sets of values of the variables. From the point of view of framework development, we do not aim at representing the mathematical relation itself but only the type of relation. The former would mean that we represent a concrete model (e.g., "Biomass $=2 *$ Precipitation"), while the latter means that we only represent which set of variables (called domain of the mathematical relation) influences which other set of variables (called co-domain of the mathematical relation). Furthermore, we give a name to each process. The process by which the variable "Precipitation" influences "Biomass", for example, could be called "Growth". In METAFRAME we represent this as:

Growth: Precipitation -> Biomass

Or if several variables are involved:

Growth: (Biomass, Precipitation) -> Biomass

\section{The refined version of the Ostrom SES framework represented in METAFRAME}

The formalization of the SES framework proposed by Ostrom (2009) - with changes as proposed by Ostrom and McGinnis (this issue) - that is discussed in Section 4 of this paper can be denoted in METAFRAME as a single concept:

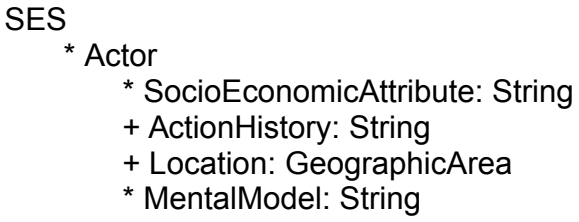

* MentalModel: String 


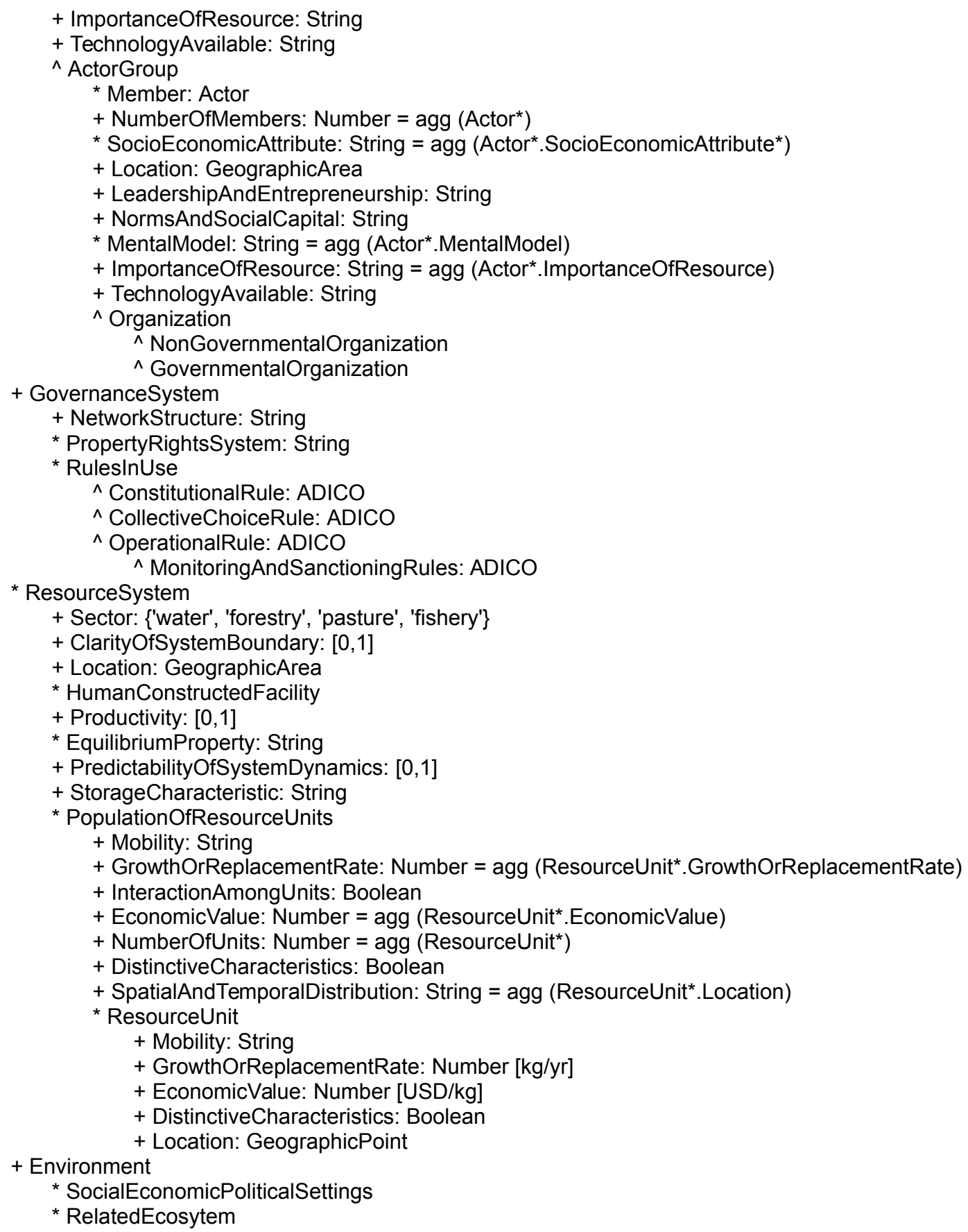

\section{Notes on design decisions:}

This formalization is by no means the definitive version of the formal SES framework. As explained in the main text of this article, framework development is a dynamic process. At any time, SES researchers may introduce new variables as part of their inquiry of a particular SES, or comparison across multiple SESs. Accommodating these new variables within the framework may lead to the definition of new concepts and/or modification of existing concepts. 


\section{ResourceSystem:}

- the value of sector (RS1) is one of a limited set of string values;

- clarity of system boundaries (RS2) and predictability of system dynamics (RS7) can be expressed on a scale from 0 (low) to 1 (high);

- equilibrium properties (RS6) and storage characteristics (RS8) are (for now) described in natural language; and

- By representing location (RS9) as a geographical area, we also include the variable RS3 (Size of resource system)

\section{ResourceUnit:}

- The name of variable RU3 (Interaction among resource units) suggests a process, and these we would formalize as process relationships.

- We decided to define "Mobility" to be of type "String", because defining it by means of an aggregation relation with Location (e.g., movement rate in $\mathrm{m} /$ day) may not capture the information that resource units are territorial, or that their movement is confined. The scale units for growth rate and economic value may also be subject to debate.

- "PopulationOfResourceUnits.GrowthOrReplacementRate" is not necessarily an aggregation of "ResourceUnits.GrowthOrReplacementRate", because in fisheries, for example, the growth rate of a population relates to an increase in numbers or biomass while the growth rate of an individual is often measured as an increase in length.

\section{Actors}

- We struggled with the alternative to see "Organization" as a direct sub-concept of "Actor" and not of "ActorGroup". While clearly an organization "is-a" special kind of actor group, it is not clear that one would want to describe the internals of an organization in terms of all ActorGroup variables such as social capital and leadership. The point is that in any analysis of SES one needs to decide which social entities to resolve and describe internally and which to treat as unresolved holistic collective actors and one could regard organizations as the latter type.

- We give "location" (A4) the data type "GeographicArea" because this offers more flexibility than single point locations.

\section{Formalization of institutions in ADICO}

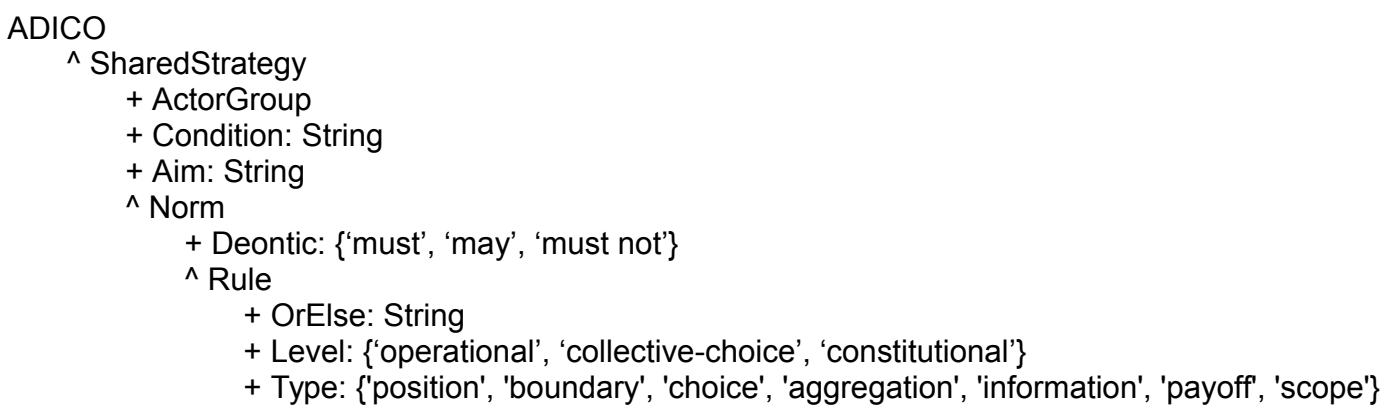

This concept hierarchy reflects that rules are the most specific form of institution. A rule specifies for a particular group of actors under which condition it must (obligation), may (permission or right), or must not (prohibition) aim for a particular goal or action, or else risk 
certain sanctions. A norm is a rule without sanctions; a shared strategy has no deontic and hence merely states under which conditions a group is willing to take collective action. Note that we have added the attribute Level to allow distinguishing between the three types of rules. 


\section{A framework of a recreational fishery case represented in METAFRAME}

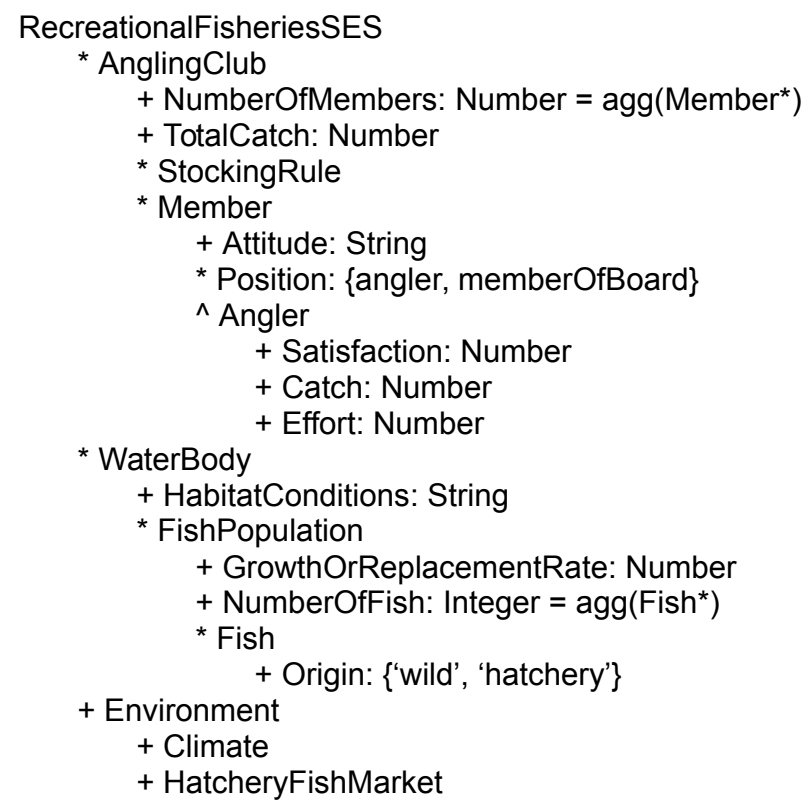




\section{The refined Ostrom framework merged with the framework of the recreational fishery case.}

This appendix shows how the Ostrom framework (Section 2) is extended with the framework of the recreational fishery case (Section 3). The black concepts/variables are those of the general SES framework that have not been directly used in the recreational fishery case, the blue concepts are those that have and the red concepts are those that have newly been added.

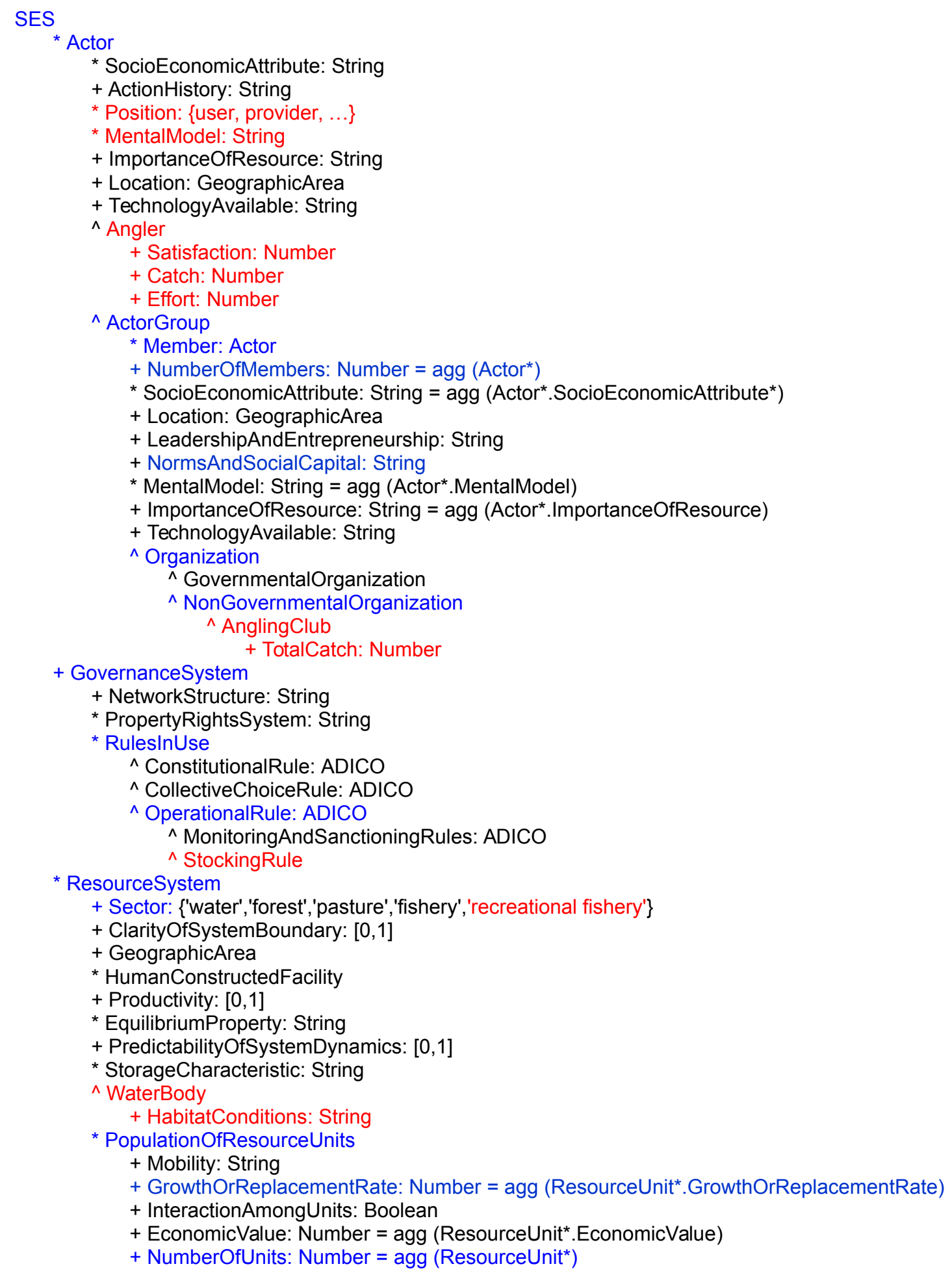


+ DistinctiveCharacteristics: Boolean

+ SpatialAndTemporalDistribution: String = agg (ResourceUnit ${ }^{*}$.Location)

${ }^{*}$ ResourceUnit

+ Mobility: String

+ GrowthOrReplacementRate: Number

+ EconomicValue: Number

+ DistinctiveCharacteristics: Boolean

+ Location: GeographicPoint

$\wedge$ Fish

+ Origin: $\{$ 'wild', 'hatchery'\}

+ Environment

* SocialEconomicPoliticalSettings

$\wedge$ HatcheryFishMarket

* RelatedEcosytem

$\wedge$ Climate 